\title{
The Spirit of Place and its Ghost.
}

\section{Edoardo Tresoldi's creative Restoration}

\author{
Alice Iacobone \\ alice.iacobone@gmail.com
}

\begin{abstract}
Christian basilica in Siponto, Apulia, realized by Italian artist Edoardo Tresoldi. Tresoldi resorts to the wire mesh as an artistic material: the restored basilica thus results in a monumental but evanescent building. After an introduction describing the public artwork, the paper will unfold in two main sections. In the first one, the focus will be on the concept of genius loci as a distinctive character of Tresoldi's poetics. Through an Heideggerian lens, the stress will be put on the existential value of dwelling, which is highlighted by the restored church. The second section will focus on the ghostly and uncanny (unheimlich) appearance of the basilica. Thanks to authors such as Derrida and Didi-Huberman, it will be shown that a genius deloci is at work in the site as well, calling into question the different temporalities that animate the basilica. Genius loci and genius deloci will prove as a conceptual couple capable of providing us with an access to the artwork; nevertheless, they will not be understood as a dichotomy, i.e. as mutually exclusive concepts, but as opposite notions that "haunt" each other and that cannot but be used together, in the postmodernist fashion of double-coding.
\end{abstract}

Keywords: Edoardo Tresoldi, Martin Heidegger, Jacques Derrida, genius loci. 


\title{
L'esprit du lieu et son fantôme. La restauration créative d'Edoardo Tresoldi
}

\author{
Alice Iacobone \\ alice.iacobone@gmail.com
}

Il faut parler $d u$ fantôme, voire au fantôme et avec lui.

J. Derrida

\section{L'église faible. Description de l’intervention à Siponto}

En 2015 le Ministère du Tourisme italien finance la restauration de la Basilique paléochrétienne de Siponto, dans les Pouilles, et confie le projet au jeune artiste Edoardo Tresoldi. Ce qui en résulte aujourd'hui est une œuvre à la fois monumentale et éthérée, dont l'aspect diaphane est la conséquence de la singulière " matière absente " adoptée par Tresoldi : le treillis soudé (fig. 1, fig. 2). La structure, haute de 14 mètres pour un poids de 7 tonnes, se dresse sur le plan de la Basilique de Santa Maria Maggiore, érigée vers 465 av. J.-C. mais détruite par une série de tremblements de terre entre le $\mathrm{XI}^{\mathrm{e}}$ et le $\mathrm{XIII}^{\mathrm{e}}$ siècle ${ }^{1}$. La conservation et la mise en valeur du site archéologique, bien qu'elles constituent des éléments centraux du projet, jouent ici un rôle qu'on ne peut comprendre qu'en considérant le dialogue entre ces aspects et le côté expressément artistique du travail : de fait, la Basilique en treillis se présente comme une intervention d'art public, tout en mettant en relation archéologie, architecture et art contemporain.

\footnotetext{
${ }^{1}$ C. Innaco, L'altra metà della Rovina. Il progetto di Edoardo Tresoldi per la Basilica di Siponto, in "PsicoArt - Rivista di arte e psicologia", IX, 2019, pp. 1-24, ici pp. 2-5; F. Del Sole, The Architectural Illusion of Edoardo Tresoldi: The Reconstruction of the Basilica of Siponto, in "Athens Journal of Architecture", VII/2, 2021, pp. 257-274, ici pp. 258-259.
} 


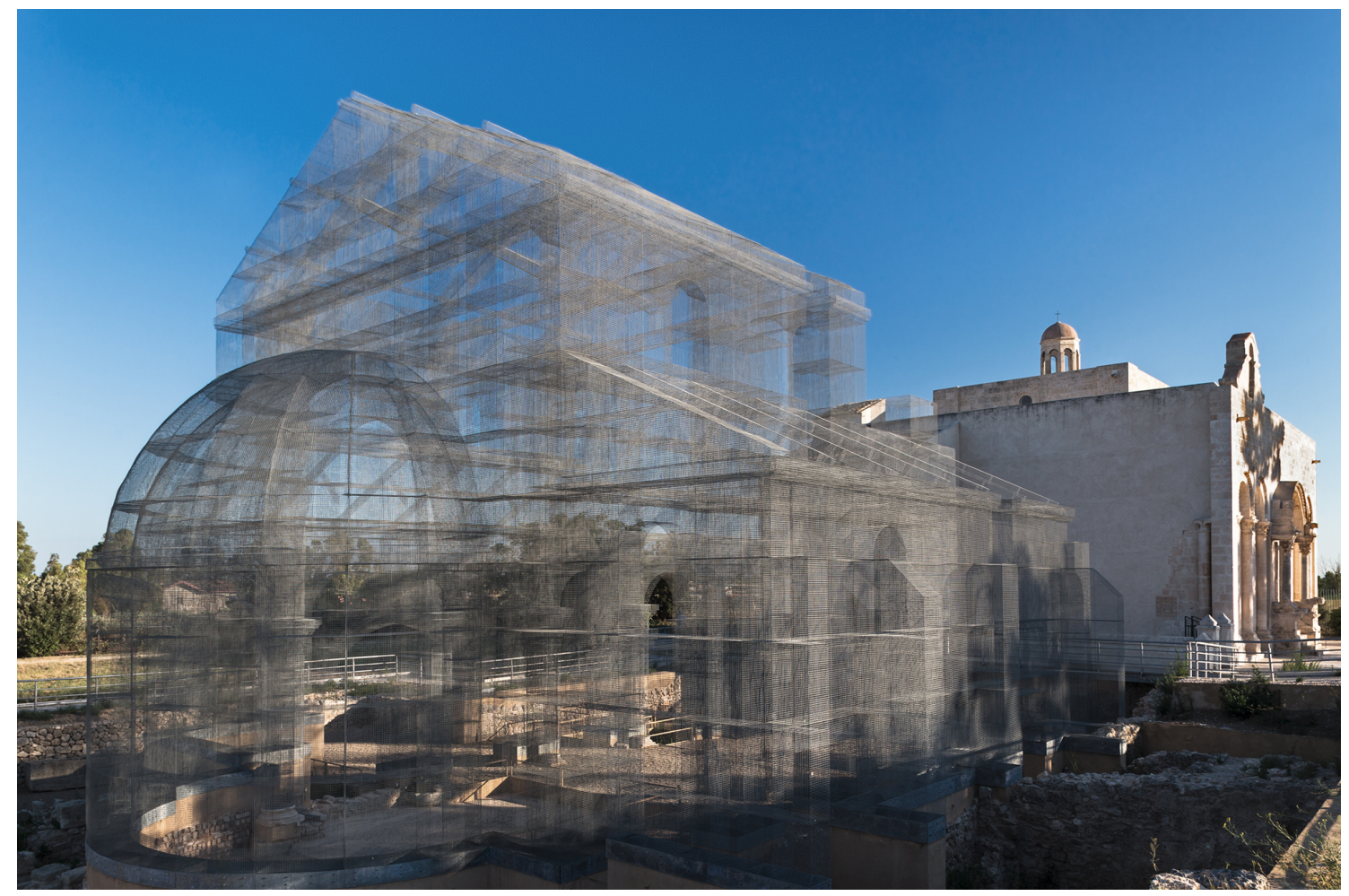

Fig. 1 Basilique de Santa Maria Maggiore restaurée par Edoardo Tresoldi, 2016, site archéologique de Siponto, Manfredonia (FG), Italie. Photographie C Roberto Conte, permission de l'artiste.

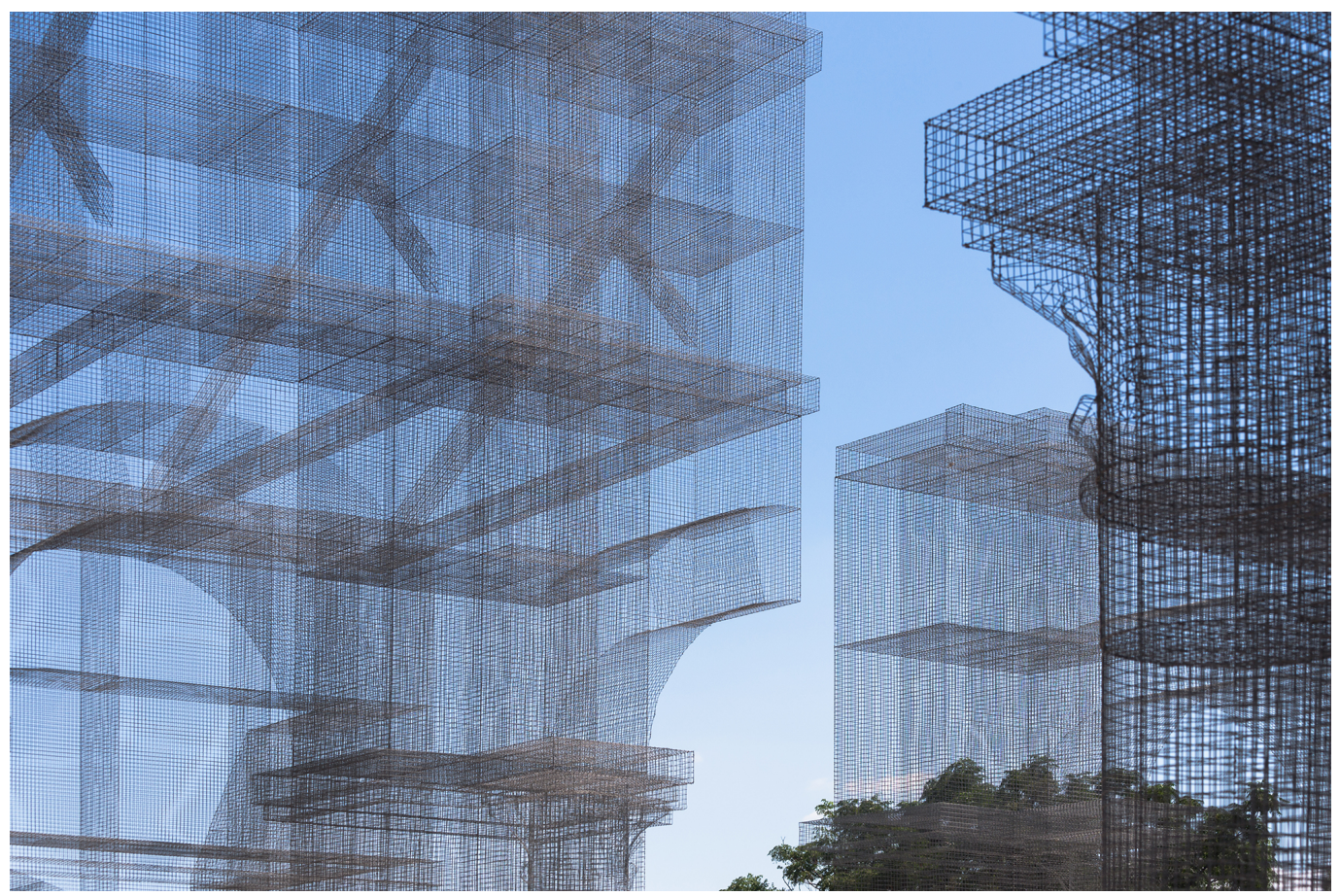

Fig. 2 Détail du treillis soudé, la "matière absente" d’Edoardo Tresoldi. Photographie (C) Roberto Conte, permission de l'artiste.

19। 
L'œuvre, pensée pour sa réalisation in situ et donc profondément intégrée à l'environnement et au paysage, respecte les éléments archéologiques du lieu dans le geste même qui conduit à les modifier : la Basilique réalisée par Tresoldi ne vise pas une ressemblance sans écart avec la Basilique originaire, mais, en renonçant à toute reconstruction mimétique, cherche à redonner vie au lieu grâce à une " ressemblance par contact" avec le bâtiment paléochrétien, sur les fondations duquel elle repose ${ }^{2}$. Comme on a pu le remarquer, «c'est de la proximité avec la matrice représentée par les traces archéologiques, de la proximité avec le lieu même où a été créée l'empreinte sur laquelle la nouvelle structure s'appuie, que naît la ressemblance entre l'installation et la Basilique paléochrétienne $»^{3}:$ dans le geste de Tresoldi la dimension philologique est à la fois considérée et détournée. En conséquence, l'opération menée à Siponto n'est ni une simple restauration de conservation ni un exemple d'anastylose : elle représente bien plutôt un cas de restauration créative ${ }^{4}$.

Le projet vise également, et de manière explicite, à souligner le rôle majeur attribué à la réception et à la figure du visiteur, ce qui met en lumière la valeur sociale de cette restauration artistique. Non seulement l'opération a pour but la promotion et la valorisation du site archéologique en éveillant l'intérêt du public, mais le public luimême se trouve impliqué par la structure de la Basilique rebâtie : tout se passe comme si la Basilique devait être habitée pour pouvoir “fonctionner". L'importance de la relation entre visiteur et installation ainsi que l'attention accordée à la dimension de l'expérience ont été soulignées par Tresoldi par le moyen de deux procédés différents. Le premier tient à la présence de sept sculptures anthropomorphes, réalisées elles-aussi en treillis métallique, qui peuplent l'œuvre avec l'évanescence fantasmatique qui caractérise l'ensemble du travail. Ces figures cherchent à suggérer aux visiteurs des perspectives sur et dans l'installation, tout en proposant des postures ou des placements qu'il faudrait concevoir d'une façon atmosphérique ou expérientielle plutôt que simplement optique ou visuelle.

\footnotetext{
${ }^{2}$ Voir G. Didi-Huberman, La ressemblance par contact. Archéologie, anachronisme et modernité de l'empreinte, Les Éditions de Minuit, Paris 2008.

${ }^{3}$ C. Innaco, L'altra metà della Rovina, cit., p. 11 (tr. de l'auteur).

${ }^{4}$ Sur le sujet d'une restauration créative (telle qu'elle avait été théorisée par Cesare Brandi) à l'œuvre chez l'intervention de Tresoldi à Siponto cf. Del Sole, The Architectural Illusion of Edoardo Tresoldi, cit.
} 
Grâce au lien empathique que l'on crée avec le personnage [avec la sculpture] - affirme Tresoldi dans une interview - le visiteur peut adopter une grille de lecture spécifique pour chercher à comprendre l'espace. À Siponto, je voulais raconter ce lieu-là : je me suis identifié au visiteur et j'ai pensé que créer, ou souligner, une intimité avec le lieu pouvait représenter une manière de « conseiller » personnellement les visiteurs ${ }^{5}$.

Parce qu'elle implique l'empreinte du corps de Tresoldi et l'adoption de ses points de vue personnels, l'introduction des sculptures peut être conçue comme une signature inscrite par l'auteur dans son œuvre ${ }^{6}$, ce qui place artiste et spectateur dans un rapport de réversibilité et de continuité. Cependant, dans la mesure où il s'agit d'une signature, on peut interpréter la présence de ces sculptures comme «trace» derridienne, voire comme diffraction de la présence de l'auteur et non pas comme revendication d'une paternité pleine, limpide, "saturée ",

Pourtant, cette première stratégie adoptée par Tresoldi afin de souligner l'importance du public dans l'œuvre semble faible si on la compare à la deuxième. L'artiste choisit de laisser inachevée la structure de l'église en treillis métallique en introduisant des déchirures dans certaines parties du bâtiment: la nef de gauche, par exemple, ne présente que les soubassements des piliers; le toit n'est rebâti que partiellement ${ }^{8}$. Tresoldi réalise ainsi une œuvre qui relève de la catégorie du non finito ou de celle des “œuvres ouvertes". De cette manière, l'artiste interpelle le visiteur en exigeant qu'il adopte une posture active - c'est-à-dire, la perception d'une structure lacunaire invite le visiteur à achever cette structure-même grâce à une activité " imaginative "9. Comme l'explique Tresoldi,

Ce qui importe - du point de vue physique et du point de vue expérientiel - c'est la possibilité d"“ éteindre " une église et d'en " allumer" une autre, en permettant au visiteur d'en retracer mentalement certaines parties tout en lui donnant des points de départ ou des points de repère. [...] Une fois que le cœur de l'église a été rebâti et que les éléments qui permettent de reconnaître la Basilique paléochrétienne ont été placés, j'ai cherché à

\footnotetext{
${ }^{5}$ C. Innaco, L'altra metà della Rovina, cit., p. 19 (tr. de l'auteur).

${ }^{6}$ Ibidem.

${ }^{7}$ J. Derrida, Signature événement contexte, in Id. Marges de la philosophie, Les Éditions de Minuit, Paris 1972, pp. 365-393.

${ }^{8}$ C. Innaco, L'altra metà della Rovina, cit., p. 13.

${ }^{9}$ Cf. F. Tononi, Aesthetic Response to the Unfinished: Empathy, Imagination and Imitation Learning, in

"Aisthesis", XIII/1, 2020.

199
}

Itinera, N. 22, 2021 
composer et décomposer les éléments restants comme un pur sculpteur, afin de dégager des espaces expressifs et de créer des tensions plutôt que des vides ${ }^{10}$.

La Basilique est donc davantage un «parcours à agir » ${ }^{11}$ qu'un objet simplement conçu pour la réception passive ou à la contemplation désintéressée. Avec ses formes, le bâtiment offre des occasions aux visiteurs, des affordances qui, comme l'affirmait Umberto Eco, invitent le spectateur à une intervention active grâce à la polyvalence des données initiales ${ }^{12}$.

Le caractère plurivoque exhibé par la structure inachevée permet d'examiner la Basilique de Tresoldi sans prétendre la ramener à l'univocité interprétative; bien au contraire, il est possible ici de mettre en évidence une dynamique postmoderniste de "double coding" $"$. Contamination et interférence entre différents langages et éléments deviennent alors des atouts ${ }^{14}: 1^{\prime}$ 'opération réalisée à Siponto peut être abordée selon des démarches et des concepts qui s'opposent les uns aux autres. C'est précisément cette implication mutuelle dissonante qui produit un sens inédit et confirme l'originalité du geste de Tresoldi. Parmi ces nombreux couples de catégories il y a l'opposition “antiquité-contemporanéité " : celle-ci se décline, par exemple, dans la différence entre l'aspect de la Basilique à la lumière du jour et son apparence nocturne, quand elle est illuminée par des lumières artificielles (fig. 3, fig. 4). On peut relever un deuxième court-circuit tout aussi fécond si l'on considère le couple représenté par la sacralité de l'église, bâtiment noble, et le treillis métallique, matériau pauvre - un court-circuit qui pourrait aisément conduire à l'idée d'une “ nouvelle monumentalité ” réalisée à l'aide de nouveaux matériaux : une «monumentalité “ faible " ", donc, qui s'impose dans le

\footnotetext{
${ }^{10}$ C. Innaco, L'altra metà della Rovina, cit., p. pp. 16-17, (tr. de l'auteur).

${ }^{11}$ Ivi, p. 11, (tr. de l'auteur).

${ }^{12}$ U. Eco, Opera aperta. Forma e indeterminazione nelle poetiche contemporanee (1962), Bompiani, Milano $2016^{10}$, p. 155.

13 "What is a typical Post-Modern building?", se demande Charles Jencks dans l'un de ses textes consacrés au postmodernisme. "One that is hybrid, one that dramatises the mixture of opposing periods - the past, present and future [...]. Hence it is based on multiple codes, combining Modern universal technology and local culture, in a recognisable "double-coding", its characteristic style » (C. A. Jencks, The Story of Post-Modernism. Five Decades of Ironic, Iconic and Critical in Architecture, Wiley, Chichester 2011, p. 11). Voir aussi le classique C. A. Jencks, The Language of Post-Modern Architecture, Academy Editions, London 1977.

${ }^{14}$ C. Innaco, L'altra metà della Rovina, cit., p. 17.
} 

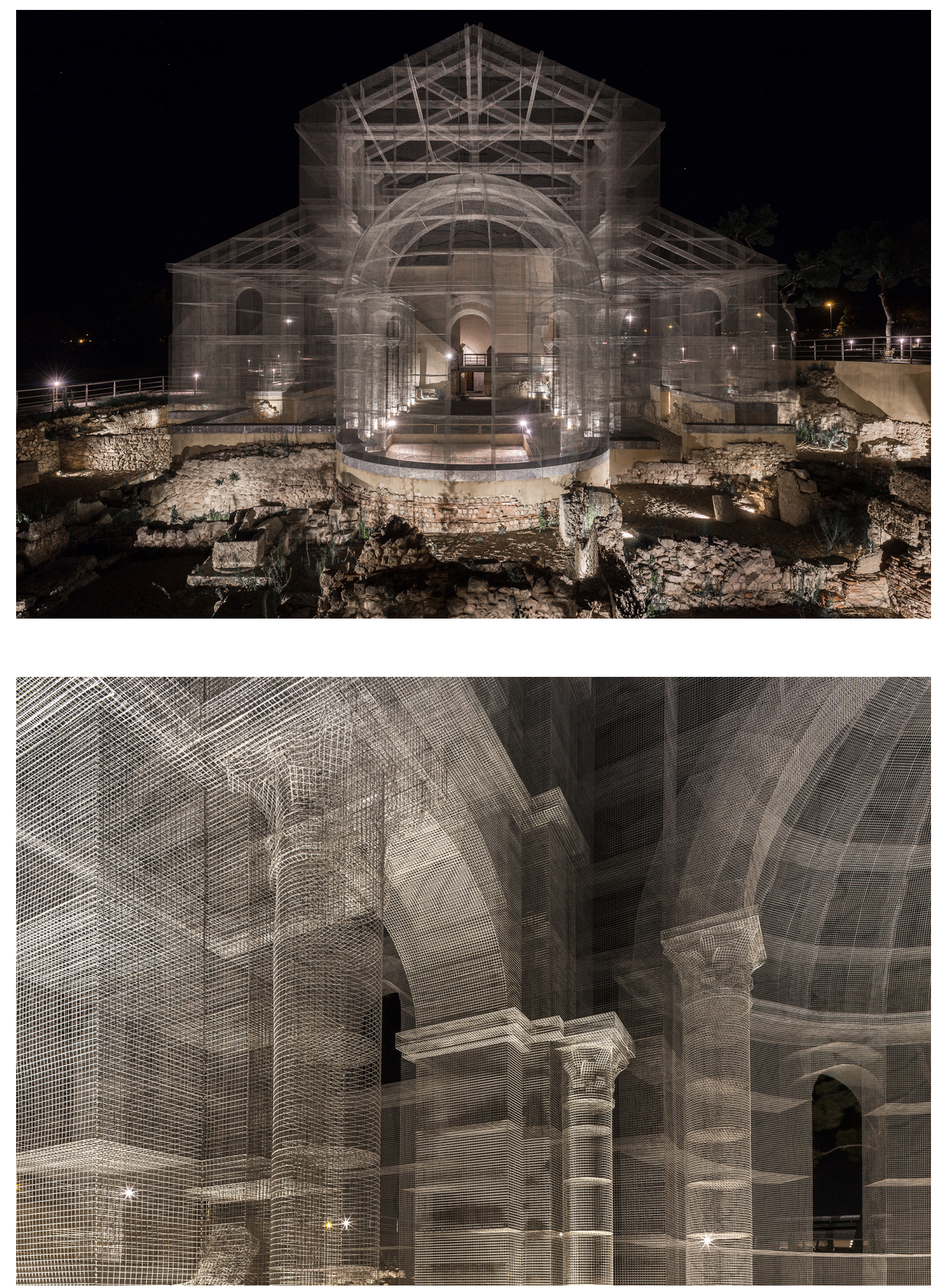

Fig. 3, fig. 4 La Basilique illuminée pendant la nuit. Photographie $\odot$ Roberto Conte, permission de l'artiste. 
mouvement même de sa disparition ${ }^{15}$. Dans ce qui suit, ce sera justement l'un de ces “doubles codes" qui nous donnera une piste à suivre afin d'aborder spéculativement le travail achevé à Siponto. On montrera ainsi que le genius loci, élément clé de la poétique de Tresoldi, s'accompagne toujours de son opposé spectral, qu'il n'y a aucune habitation sans dépaysement, qu'il n'y a aucune fondation sans retrait du fondement et que la Basilique de Tresoldi incarne l'esprit du lieu précisément quand elle laisse transparaître, à travers ses mailles métalliques, le fantôme qui la hante.

\section{Habiter ce qui reste. Présences du genius loci}

Bien que le style de Tresoldi se caractérise par l'emploi du treillis métallique, il ne paraît pas exagéré d'affirmer que la caractéristique majeure de sa poétique réside dans l'attention consacrée à la notion antique de genius loci, qu'on rencontre souvent au fil de ses déclarations. De fait, né dans la Rome antique, le concept a trouvé une large réception dans le débat sur l'architecture et l'urbanisme qui s'est développé en Italie dans les années soixante et soixante-dix ${ }^{16}$, période où on s'éloignait du fonctionnalisme afin de renverser la tendance rationaliste du modernisme pour mettre l'accent sur la dimension existentielle et locale de l'habiter. L"“ esprit du lieu " ne révèle pas ce qu'est le lieu depuis toujours, il montre plutôt son «caractère », ce qu'il veut être ${ }^{17}$. Le genius loci serait donc l'identité du lieu en tant qu'essence dynamique, toujours prise dans le devenir engagé par la relation avec le milieu; mais le concept évoque aussi, réciproquement, l'instauration d'identités locales et plurielles pour ceux qui demeurent dans ce lieu-là. On a pu remarquer qu' « en faisant-lieu on assiste à une co-production et à une coévolution du sens des lieux et des identités de ceux qui les habitent, traversent,

\footnotetext{
15 A. Vidler, The Architectural Uncanny. Essays in the Modern Unhomely, Massachussets Institute of Technology, Cambridge (MA) 1992, p. 220 (tr. de l'auteur).

${ }^{16}$ Voir, par exemple, A. Rossi, L'architettura della città, Marsilio, Padova 1966 ou F. Purini, Luogo e progetto, Magma, Roma 1976. Le débat architectural italien sur le genius loci s'est poursuivi jusqu'à aujourd'hui. Il suffit en effet de mentionner la revue «Sensibilia » qui a dédié un numéro monographique à ce thème (vol. 9, éd. par S. Pedroni et M. Tedeschini, 2015). De même, en 2021 on note la parution d'un texte de Stefano Chiodi où le concept de genius loci devient une notion centrale dans la reconstruction des relations ambivalentes de l'art italien avec les procédés d'autoreconnaissance culturelle et politique (S. Chiodi, Genius loci. Anatomia di un mito italiano, Quodlibet, Macerata 2021).

17 C. Norberg-Schulz, Genius Loci. Paysage Ambience Architecture, traduit par O. Seyler, Mardaga éditeur, Sprimont 1981, p. 18.
} 
abandonnent $\gg{ }^{18}$. En ce qui concerne le projet de Siponto, le discours sur l'esprit du lieu a été déployé surtout à travers l'analyse des relations qui s'activent entre le bâtiment et le paysage dans lequel l'ouvrage est plongé. On peut évoquer par exemple la relation productive instaurée entre l'abside de la Basilique et l'arbre qui se trouve derrière elle : un Pinus halepensis, espèce centenaire qui caractérise la région du Gargano, s'inscrit dans l'image de l'abside grâce au jeu de transparences créé par le treillis métallique ; en prenant la place traditionnellement occupée par l'autel et le tabernacle eucharistique ${ }^{19}$, la plante suggère une sacralité pour ainsi dire arborescente, une sacralité qui se détache donc du plan étroitement religieux et de son sens anthropique (fig. 5, fig. 6).

L'attention portée dans la deuxième moitié du vingtième siècle au concept de genius loci, dont la singularité doit être conçue comme le produit des interactions hétérogènes qui traversent et façonnent le lieu diachroniquement et synchroniquement, s'exprime notamment dans un texte écrit par Christian Norberg-Schulz ${ }^{20}$. En adoptant une optique heideggerienne, l'auteur y développe une phénoménologie de l'architecture qui se déploie autour de la relation existentielle entre l'être humain et son milieu. Dans ce cadre-là, l'architecture «visualise $»^{21}$, voire «concrétise $»^{22}$ l'esprit du lieu, en l'incarnant. La première tâche du bâtiment consiste à «comprendre la "vocation" du lieu $»^{23}$; néanmoins, cela n'implique pas « une sorte de "déterminisme du milieu " ${ }^{24}$ car l'agency humaine est déjà co-impliquée et intégrée au lieu-même : l'agentivité humaine façonne le lieu et en est façonnée à son tour. La construction d'identité passe par un procédé au fil duquel on devient “ amis" d'un milieu donné. « Jede Stimmung ist Übereinstimmung ${ }^{25}:$ ' 'atmosphère du lieu, voire son esprit ${ }^{26}$, ne se donne que dans la correspondance entre être humain et milieu.

\footnotetext{
${ }^{18}$ C. Grasseni, Luoghi comuni. Antropologia dei luoghi e pratiche della visione, Lubrina, Bergamo 2009, p. 15 (tr. de l'auteur).

${ }^{19}$ C. Innaco, L'altra metà della Rovina, cit., p. 13, p. 18.

${ }^{20}$ C. Norberg-Schulz, Genius Loci, cit.

${ }^{21}$ Ibidem.

${ }^{22}$ Ivi, p. 23.

${ }^{23}$ Ibidem.

${ }^{24}$ Ibidem.

${ }^{25}$ L'expression, par Otto Friedrich Bollnow, est citée par Norberg-Schulz in $i v i$, p. 21.

${ }^{26}$ Le lien entre genius loci et atmosphère a été relevé par Tonino Griffero, selon lequel « au moment où l'espace acquiert une puissance atmosphérique, il émane un genius loci» (T. Griffero, Atmosferologia. Estetica degli spazi emozionali, Laterza, Roma-Bari 2013, p. 81; tr. de 1'auteur); la liaison entre atmosphère, genius loci et architecture a été soulignée aussi par G. Böhme, T. Griffero, J.P. Thibaud, Architecture and Atmosphere, Tapio Wirkkala Rut Bryk Foundation, Espoo 2014.
} 203 

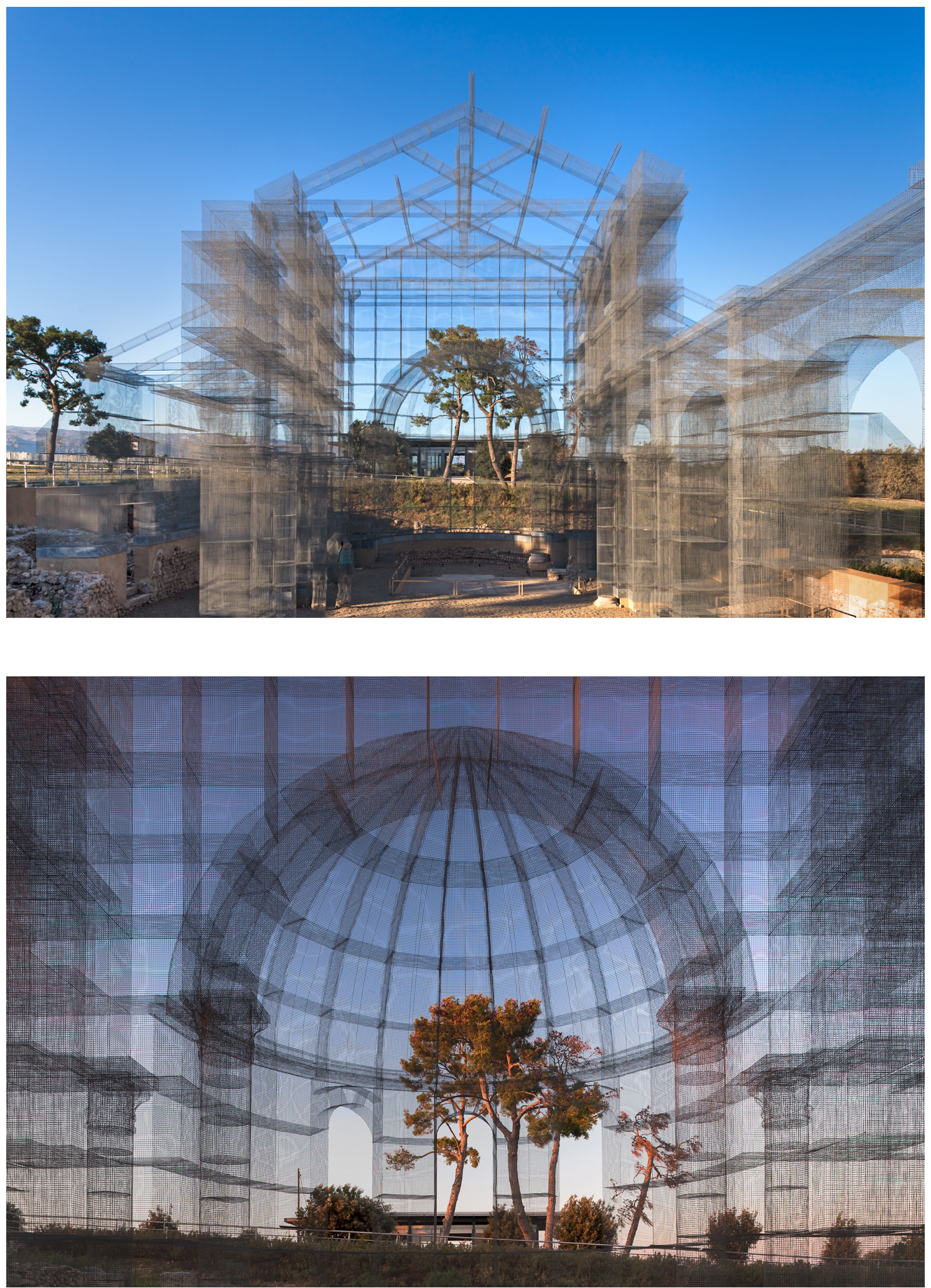

Fig. 5, fig. 6 Le pin d'Alep inséré par transparence dans l'abside de l'église. Photographie (C) Roberto Conte, permission de l'artiste. 
Le genius loci exprime la co-implication entre être humain et milieu et s'incarne dans l'élément architectural. Dans cette perspective, l'architecture «aide l'homme à habiter $»^{27}$; le bâtiment créée une jonction entre être humain et espace, donnant lieu au lieu. L'habiter, «nature de notre espèce $»^{28}$, a été l'objet d'une célèbre conférence donnée par Martin Heidegger en août 1951 (Bauen Wohnen Denken). D'après Heidegger, il faudrait placer la dimension existentielle avant toute construction, dans le sens où il faudrait que le déploiement rationaliste et abstrait de l'espace soit précédé de la “ prise existentielle" du demeurer. Comme l'écrit Heidegger :

\begin{abstract}
Le rapport de l'homme à des lieux et, par des lieux, à des espaces réside dans l'habitation.
La relation de l'homme et de l'espace n'est rien d'autre que l'habitation pensée dans son être. [...] Bâtir est, dans son être, faire habiter. Réaliser l'être du bâtir, c'est édifier des lieux par l'assemblement de leurs espaces. C'est seulement quand nous pouvons habiter que nous pouvons bâtir ${ }^{29}$.
\end{abstract}

L'œuvre de Tresoldi et sa poétique centrée sur la notion de genius loci se rapprochent beaucoup de cette perspective, surtout si l'on considère que l'architecture de l'artiste naît " déjà habitée " - puisque le visiteur est impliqué dès le début dans la constitution même de l'installation. À cet égard, on peut évoquer certaines réflexions de Gianni Vattimo à propos du statut et des modalités de la réception artistique. Le philosophe, en développant des thèses heideggeriennes, affirme que la réception se définit comme «un demeurer au sein de l'ouverture ouverte par l'œuvre, voire dans le monde qu'elle installe $»^{30}:$ l'activité du visiteur se configure en tant qu'habitation auprès de l'œuvre. Il semblerait donc que la Basilique de Tresoldi, conformément à la pensée de Heidegger, ne fasse pas partie d'un monde, qu'elle ne représente pas quelque chose de donné, mais, plutôt, qu'elle installe (stellt auf) un monde : qu'elle ouvre un nouvel horizon de sens auprès duquel le visiteur peut, à son tour, s'installer.

\footnotetext{
${ }^{27}$ C. Norberg-Schulz, Genius Loci, cit., p. 5.

${ }^{28}$ M. Vitta, Dell'abitare. Corpi spazi oggetti immagini, Einaudi, Torino 2008, p. 3 (tr. de l'auteur).

${ }^{29}$ M. Heidegger, Bâtir habiter penser (1951), in Id. Essais et conférences, traduit par A. Préau, Gallimard, Paris 1958, pp. 170-193, ici pp. 188-191.

${ }^{30}$ G. Vattimo, Poesia e ontologia, Mursia, Milano 1967, p. 124 (tr. de l'auteur).
} 205 
Toutefois, cette perspective sur la restauration de la Basilique de Siponto apparaît beaucoup trop univoque et irénique. Heidegger lui-même pourrait nous conduire à une lecture bien plus stratifiée que celle-ci. Le monde, principe positif d'ouverture, se fonde en effet sur la terre ${ }^{31}$, fond sans fond, abîme qui se retire sans cesse. D'ailleurs, ce n'est que dans sa pensée tardive que Heidegger, de façon nostalgique, met l'accent sur une possibilité renouvelée d'enracinement. Mais si on pense à d'autres textes plus anciens, il n'est pas difficile de repérer une conception bien différente du demeurer. Dans Etre et temps, par exemple, l'être humain est toujours et originellement hors-de-chez-soi, la condition humaine se caractérise comme un état de Unzuhause ${ }^{32}$. Déjà dans le cours tenu à Marburg en 1925, Heidegger avait parlé de dépaysement (Unheimlichkeit) comme d'un des traits majeurs du Dasein ${ }^{33}$. Chez le philosophe, la confrontation avec l'œuvre d'art est marquée aussi par une expérience dépaysante: celle du Sto $\beta^{34}$. Comparé par Vattimo au choc de Walter Benjamin ${ }^{35}$, le Stoß heideggerien nomme en effet le choc qui suspend l'évidence du quotidien, du connu, du domestique, en émancipant l'art, en même temps, de toute description univoque et conciliante. Face à cette tension entre habitation et dépaysement, on a pu remarquer que «la pensée de Heidegger [...] tourne autour d'un paradoxe ou d'une terrible ironie : l'être humain se qualifie comme être-au-monde et habiter sur terre - et pourtant nous ne sommes jamais chez nous dans le monde, jamais enracinés dans la terre $»^{36}$.

En fait, il semble possible d'affirmer qu'il ne s'agit pas d'un paradoxe, mais plutôt d'une double série d'éléments (Monde-Terre, habiter-dépayser, heimlich-unheimlich) qui, au lieu de s'exclure mutuellement et d'engendrer une opposition dialectique, s'enveloppent réciproquement selon une logique "hantologique". On verra que l'habiter ne se comprend qu'à la condition de repérer dans l'habitation les traits troublants et spectraux de l'Unheimliche, de la perte du monde, et vice versa : la hantise devient alors structure-même de la pensée, car chaque notion se révèle hantée par son

\footnotetext{
${ }^{31}$ M. Heidegger, L'origine de l'œuvre d'art (1936), in Id. Chemins qui ne mènent nulle part, traduit par W. Brokmeier, Gallimard, Paris 1962, pp. 7-68, ici p. 35.

${ }^{32}$ M. Heidegger, Etre et temps (1927), traduit par F. Vezin, Gallimard, Paris 1986, § 40.

${ }^{33}$ M. Heidegger, Prolégomènes à l'histoire du concept de temps (1925), traduit par A. Boutot, Gallimard, Paris 2006.

${ }^{34} \mathrm{M}$. Heidegger, L'origine de l'œuvre d'art, cit., pp. 49-50.

${ }^{35}$ G. Vattimo, L'arte dell'oscillazione, in Id. La società trasparente (1989), Garzanti, Milano $2011^{3}$.

${ }^{36}$ D. Farrell Krell, Das Unheimliche: Architectural Sections of Heidegger and Freud, in " Research in Phenomenology ", XXII/1, 1992, pp. 43-61, ici p. 45 (tr. de l'auteur).
} 
propre contraire. Aborder la Basilique de Tresoldi à travers le prisme de la hantise entraîne l'instauration d'une tension entre l'église rebâtie et les forces centrifuges de son archéologie et de son avenir. C'est au sein de ces forces destructrices et "fantomatiques" qu'on pourra enfin reconnaître l'autre face du genius loci, esprit spectral qui va nous montrer son identité diffractée et toujours hors-temps.

\section{Spectres de l'habiter. Le pouvoir du genius deloci}

Comment peut-il arriver que l'esprit du lieu se transforme en un revenant inquiétant? Ne s'agit-il pas plutôt de découvrir que le lieu en tant que tel est depuis toujours hanté par les spectres de ses temporalités hétérogènes, par ses anachronismes ? Revenons à Tresoldi et à son trait distinctif: le treillis soudé. L'adoption de ce matériau engage deux ordres de considérations au minimum : d'une part, il est possible d'analyser le statut ontologique du treillis métallique et son fonctionnement en tant que matériau artistique ; de l'autre, on peut se pencher sur les modalités phénoménologiques de son apparaître. Structure décentralisée et acéphale par excellence, le treillis évoque de manière immédiate le réseau, espace où le renvoi se relance à l'infini, espace dans lequel la présence est toujours distribuée. C'est l'aspect le plus souligné par Tresoldi : ses installations ne sont jamais complètement présentes, ce qui est assuré par l'emploi de ce matériau. «Matière absente $»^{37}$, le treillis semble se composer d'absence plutôt que de présence, ou, plus exactement : la présence même, en se distribuant, se trouve différée et hantée par l'absence. En profitant des intervalles entre les mailles comme matériau peu conventionnel, dans l'œuvre de Tresoldi l'absence s'entrelace avec la présence dans une Verflechtung ou un entanglement et met ainsi en évidence leur inséparabilité ontologique. Du point de vue phénoménal, cette ambiguïté ontologique se traduit par la qualité diaphane du bâtiment, qui acquiert une apparence spectrale et inquiétante : la Basilique est toujours en train d'apparaître, l'installation prend un aspect virtuel grâce auquel l'église ressemble à « un hologramme projeté sur le site » ${ }^{38}$. Face à cette structure monumentale et éthérée, on fait l'expérience de quelque chose qui ressemble au Sto $\beta$ de Heidegger : dans une inversion perceptive, c'est l'entre-deux qui

\footnotetext{
${ }^{37}$ C. Innaco, L'altra metà della Rovina, cit., p. 15.

${ }^{38}$ L. Wong, Adaptive Reuse. Extending the Lives of Buildings, Birkhäuser, Basel 2017, p. 236. 207
} 
devient matière, mais une matière raréfiée et imprégnée d'absence. L'affirmation de Maurice Merlau-Ponty selon laquelle « l'aspect du monde pour nous serait bouleversé si nous réussissions à voir comme choses les intervalles entre les choses [...] et réciproquement comme fond les choses elles-mêmes $\gg{ }^{39}$ est ici fort éclairante.

Grâce au treillis métallique, Tresoldi réalise un bâtiment qui apparaît comme le fantôme d'une église : c'est justement cette reconnaissance différée qui accorde à l'œuvre une inquiétante étrangeté. Si l'habiter et ses traits iréniques sont mis expressément en évidence, ce sera précisément parmi ces éléments-là qu'il faudra rechercher les raisons implicites du dépaysement. Considérons le fait que Sigmund Freud n'a approché la définition de unheimlich que par l'analyse du terme heimlich, son contraire apparent. Au fils de certaines pages très détaillées, au cours desquelles l'inquiétante étrangeté n'est pas simplement examinée mais aussi exhibée par son écriture, Freud présente une analyse lexicographique qui le conduit à établir que " "heimlich" est un mot dont le sens se développe vers une ambivalence, jusqu'à ce qu'enfin il rencontre son contraire " unheimlich". "Unheimlich" est, d'une manière quelconque, un genre de "heimlich" " ${ }^{40}$. De même que, comme on l'a dit, l'architecture incarne le genius loci en lui donnant corps, l'Unheimlichkeit possède un lien privilégié avec la dimension architecturale. Depuis ses origines romantiques, le concept $d$ " inquiétante étrangeté " a trouvé sa place auprès des lieux dévoués à l'habiter: des maisons hantées chez E.T.A. Hoffmann à la Maison occupée de Julio Cortázar, on peut aisément déceler une tendance de la Unheimlichkeit à s'installer au sein de ce qui, en architecture, est heimlich, exhibant (et non pas incarnant) ainsi le visage spectral du genius loci. Le spectral ou le fantomatique sont immédiatement impliqués par la rencontre entre inquiétante étrangeté et architecture. C'est ce que Freud remarquait quand il disait que «[les] langues modernes ne peuvent rendre [...] l'expression [allemande] "une maison unheimlich" autrement que par cette circonlocution : une maison hantée ["a haunted house", "una casa infestata dagli spettri"] $»^{41}$; d'ailleurs, Derrida a remarqué qu' «il y aurait une coextensivité de la

\footnotetext{
${ }^{39}$ M. Merleau-Ponty, Le cinéma et la nouvelle psychologie, in “ Les Temps Modernes ”, XXVI, 1947, pp. 930-943.

${ }^{40}$ S. Freud, L'inquiétante étrangeté (1919), in Id. Essais de psychanalyse appliquée, traduit par M. Bonaparte et E. Marty, Gallimard, Paris 1933, pp. 163-210, ici p. 178.

${ }^{41}$ Ivi, p. 193.
} 
Unheimlichkeit avec l'expérience de la spectralité ${ }^{42}$. Le spectre est tout d'abord un revenant: un retour et une répétition, l'apparition de quelque chose qui est constitutivement hors de son temps.

La Basilique de Tresoldi n'est pas simplement la concrétion d'un paisible " caractère du lieu" : elle est aussi le fantôme unheimlich de l'église paléochrétienne qu'elle imite, bien qu'en absence d'un référent : elle est le revenant qui, en revenant, montre qu'il n'a jamais été présent. Ainsi que le dit l'artiste, « la matière absente entame des dialogues [...] avec l'espace et l'histoire, en projetant la substance de l'objet dans une extension temporelle inédite ; ce qui a disparu, ou n'a jamais existé, revit dans un temps qui n'est pas le sien $\gg{ }^{43}$. Il ne s'agit donc pas simplement d'une densité symbolique et iconographique : bien plus radicalement, la Basilique de Tresoldi convoque dans le lieu toute une série de temporalités hétérogènes qui se greffent les unes sur les autres et se hantent mutuellement. En suivant une logique qui rappelle celle du Nachleben d'Aby Warburg, le passé paléochrétien du bâtiment survit là où en même temps réside déjà son avenir. Une fois de plus, c'est le treillis métallique qui permet d'atteindre ce résultat: dans sa fragilité sans lyrisme, il évoque les ruines d'un avenir post-industriel, ce qui laisse imaginer quelque chose comme une " archéologie de l'avenir » ${ }^{44}$. « Je suis très curieux de savoir ce qui va se passer dans l'histoire de ce monument, qui nait déjà comme le fantôme d'un monument qui n'existe plus » ${ }^{45}$, déclare l'artiste. Ce n'est pas seulement le passé qui devient fantomatique, mais également l'avenir, surtout si on considère que cette œuvre d'art ne peut jamais se comprendre en tant qu'entièrement "présente". Ce «montage de temps hétérogènes», cette «composition d'anachronismes ${ }^{46}$ fait que la Basilique de Tresoldi ne peut pas s'insérer dans une temporalité linéaire : elle invite plutôt à une manière différente d'envisager le temps. Il s'agit d'un temps qui, comme le dit Derrida, «est hors de ses gonds »: un temps

\footnotetext{
${ }^{42}$ J. Derrida, Répondre du Secret, inédit, 1991/1992. Séminaire tenu à l'Ecole des Hautes Etudes en Sciences Sociales (EHESS), conservé à l'Institut Mémoire de l'Edition Contemporaine (IMEC).

${ }^{43}$ C. Innaco, L'altra metà della Rovina, cit., p. 15 (tr. de l'auteur).

${ }^{44}$ L'expression est tirée par F. Jameson, Archaeologies of the Future: The Desire Called Utopia and other Science Fictions, Verso, London 2005 ; mais cela a donné des résultats intéressants aussi pour ce qui concerne le lien entre les arts et le souci écologique. Cf. par exemple R. Aebersold, L'archéologie de l'avenir, in "Les nouvelles de l'archéologie ”, CLI, 2018, p. 71.

${ }^{45}$ C. Innaco, L'altra metà della Rovina, cit., p. 21 (tr. de l'auteur).

${ }^{46}$ G. Didi-Huberman, Génie du non-lieu. Air, poussière, empreinte, hantise, Les Éditions de Minuit, Paris 2001, p. 39. 
" détraqué », « désarticulé », « désajusté » ${ }^{47}$. C'est précisément au sein de cette disjointure temporelle qu'on repère, d'après Derrida, la possibilité d'une justice qui ne se réduise pas à la simple symétrie : c'est au moment où le temps est out of joint, c'està-dire dans une relation toujours dissymétrique avec les spectres, qu'une «politique de la mémoire, de l'héritage et des générations $»^{48}$ devient vraiment possible. Tout comme les Pathosformeln dont parle Warburg - formules qui font toujours l'objet d'une reprise créative et jamais d'une banale répétition -, le revenant met en scène une répétition qui est en même temps une première fois. Voilà donc « la question de l'événement comme question du fantôme ${ }^{49}$. «Question de répétition : un spectre est toujours un revenant. On ne saurait en contrôler les allées et venues parce qu'il commence par revenir ${ }^{50}$. L'itération performative affichée par la Basilique en treillis métallique, répétition sans terme répété, lieu du dépaysement de l'histoire, s'impose à nous en tant qu'héritage : un héritage qui «n'est jamais un donné, c'est toujours une tâche. Elle reste devant nous, aussi incontestablement que, avant même de le vouloir ou de le refuser, nous sommes des héritiers, et des héritiers endeuillés, comme tous les héritiers $»^{51}$. En prévenant l'alliance paralysante entre patrimonialisation et présentisme ${ }^{52}$, Tresoldi distribue et dissémine l'identité de la Basilique dans des temporalités variées et sans synthèse possible, en nous donnant la tâche, impossible, d'hériter de l'église : de faire face à sa présence et à son absence, en même temps.

Georges Didi-Huberman a abordé le thème du rapport entre hantise et lieu en s'appuyant sur l'idée d'un « genius deloci », voire d'un « génie du non-lieu » ${ }^{53}$ :

"Génie du non-lieu" : pouvoir réciproque de la hantise sur le lieu (il le met en mouvement [...]) et du lieu sur la hantise (il la reconfigure en lui donnant un champ d'action physique). La hantise comme lieu (elle devient quelque chose de plus qu'un esprit) instaure le lieu comme hantise (il devient quelque chose de plus qu'un espace) $)^{54}$.

\footnotetext{
${ }^{47}$ J. Derrida, Spectres de Marx, Éditions Galilée, Paris 1993, p. 42.

${ }^{4}$ Ivi, p. 15.

${ }^{49}$ Ivi, p. 31.

${ }^{50} \mathrm{Ivi}, \mathrm{p} .32$.

${ }^{51}$ Ivi, p. 94.

${ }^{52}$ Voir F. Hartog, Régimes d'historicité. Présentisme et expériences du temps, Seuil, Paris 2003.

${ }^{53}$ G. Didi-Huberman, Génie du non-lieu, cit., p. 126 et passim.

${ }^{54}$ Ivi, p. 136.
} 
Opposé, apparemment, au genius loci, cette figure montre bien comment les deux pôles du discours ne s'excluent pas mutuellement mais procèdent au contraire par involution réciproque. Le genius deloci n'est pas exclusivement dislocation, car lui aussi se montre capable d'instaurer un lieu. L'esprit du lieu et son fantôme modifient l'espace, le métamorphosent en en faisant, précisément, un locus. Ce n'est donc que dans le rapport indissoluble du genius loci et du genius deloci que le lieu se donne en héritage, en évitant à la fois de rester paralysé en identité immuable, lumineuse mais mortifère, et de se disperser dans une multitude fantomatique, dans une dissémination sans ancrage.

\section{Conclusion}

« L'œuvre n'imite pas un espace. Elle produit son lieu » ${ }^{55}$. Cette expression de Georges Didi-Huberman décrit parfaitement le sens de l'opération menée à Siponto par Tresoldi. Celle-ci met en place deux stratégies, différentes et pourtant indissociables : d'un côté, «l'œuvre $[. .$.$] produit son lieu » { }^{56}$ dans la mesure où l'œuvre d'art installe un monde et incarne le genius loci, en lui donnant corps, forme, identité, ce qui assure habitabilité et enracinement. De l'autre, le simple espace devient lieu également grâce aux fantômes inquiétants qui le hantent, revenants de son passé mais aussi de son avenir : «la hantise métamorphose l'espace - donnant naissance à un lieu ${ }^{57}$. Il s'agit du double code à travers lequel il faut considérer la Basilique de Tresoldi : intimement impliquée dans la relation avec ses visiteurs et, en même temps, inquiétante et sombrement hostile; expression physique et matérielle du genius loci, tout en étant une présentation éminente du caractère disloqué et manquant d'un genius deloci qui ne peut jamais ni s'incarner ni être localisé (que ce soit temporellement ou topographiquement) ${ }^{58}$. Le concept de genius deloci ne vise pas à offrir une alternative à la notion de genius loci. Les deux concepts n'entrent pas dans un rapport dichotomique : disons plutôt que l'un est une variante de l'autre. Le fantôme peut toujours se renverser en esprit du lieu, et, réciproquement, c'est quand on se trouve face aux éléments les plus familiers qu'il

\footnotetext{
${ }^{55}$ Ivi, p. 39.

${ }^{56}$ Ibid.

${ }^{57} I v i$, p. 142.

${ }^{58}$ Le point a bien été souligné par Merleau-Ponty: «En cherchant à placer [le] fantôme, [on] le fera disparaître comme fantôme » (cité par Didi-Huberman in ivi, p. 131). Le fantôme est toujours disloqué : "Acceptons la condition défective du genius deloci », écrit Didi-Huberman, «le caractère fatalement déplacé de sa forme spectrale » (ivi, p. 131).

211
}

Itinera, N. 22, 2021 
arrive de s'exclamer, tout à coup, “es spukt”, “ça spectre ",59. Grâce à l'usage savant du treillis soudé sur une échelle monumentale, la Basilique de Tresoldi nous invite à habiter là où le lieu est traversé par la hantise de ses anachronismes. Faisant cela, elle sollicite notre responsabilité à l'égard de l'église rebâtie : la responsabilité d'un héritage impur, seul héritage possible.

${ }^{59}$ J. Derrida, Répondre du secret, cit. ; Id, Spectres de Marx, cit., p. 215. 